\title{
Experimental EPR-steering using Bell-local states
}

\author{
D. J. Saunders ${ }^{1,2}$, S. J. Jones ${ }^{1,2}$, H. M. Wiseman ${ }^{1,2 \star}$ and G. J. Pryde ${ }^{1,2 \star}$
}

The concept of 'steering' was introduced in 1935 by Schrödinger ${ }^{1}$ as a generalization of the EPR (EinsteinPodolsky-Rosen) paradox. It has recently been formalized as a quantum-information task with arbitrary bipartite states and measurements ${ }^{2}$, for which the existence of entanglement is necessary but not sufficient. Previous experiments in this area $^{3-6}$ have been restricted to an approach ${ }^{7}$ that followed the original EPR argument in considering only two different measurement settings per side. Here we demonstrate experimentally that EPR-steering occurs for mixed entangled states that are Bell local (that is, that cannot possibly demonstrate Bell non-locality). Unlike the case of Bell inequalities ${ }^{8-11}$, increasing the number of measurement settings beyond two-we use up to six-significantly increases the robustness of the EPR-steering phenomenon to noise.

It was Einstein, Podolsky and Rosen who first highlighted the fact that 'as a consequence of two different measurements performed upon the first system, the second system may be left in states with two different [kinds of] wavefunctions'12. For them, this spooky action at a distance was unacceptable, and proved that the Copenhagen interpretation of quantum mechanics was incomplete. In the example they used to illustrate this 'paradox', the two different kinds of wavefunction were position and momentum eigenstates, which are clearly incompatible because 'precise knowledge of $[Q]$ precludes such a knowledge of $[P]$ ' (ref. 12). In this paradigm, Reid $^{7}$ first developed quantitative criteria for the experimental demonstration of the EPR paradox on the basis of Heisenberg's uncertainty relation $(\Delta P)(\Delta Q) \geq \hbar / 2$; see also ref. 13 .

In the same year as the EPR paper, Schrödinger introduced the term 'steering' to describe the EPR paradox, and generalized it to more than two measurements, saying 'Since I can predict either $[Q]$ or $[P]$ without interfering with [the second] system,... [it] must know both answers; which is an amazing knowledge. [The second system] does not only know these two answers but a vast number of others'. It is only very recently that general EPR-steering inequalities, allowing for measurements of an arbitrary number of different observables by the two parties, have been developed ${ }^{14}$. This followed the first formal definition of steering in refs 2,15, which proved that demonstrating EPRsteering is strictly easier than demonstrating Bell non-locality (that is, violating a Bell inequality) but strictly harder than demonstrating non-separability (by quantum state tomography or entanglement witnesses ${ }^{16}$ ). The existence of this hierarchy is a logical consequence of the definitions, and its strictness was proved by consideration of two-qubit Werner states and restriction to projective measurements. Although non-projective measurements could, in theory, be more powerful ${ }^{17}$, this is a remote possibility for such a simple state, and we restrict ourselves to projective measurements here also. Finally, we emphasize that none of these forms of quantum non-locality allows for the possibility of faster-than-light signalling.

Here we exploit the modern formulation of EPR-steering for the first time, demonstrating EPR-steering with discrete binaryoutcome measurements on Werner states ${ }^{18}$ of a pair of photonpolarization qubits. This family of states (singlets with isotropic noise) is well studied, and it is proven that some of the states we use to demonstrate EPR-steering violate no Bell inequality. This is not the case for any of the states used in previous demonstrations ${ }^{3-6}$ of the EPR paradox, which relied on the EPR-Reid inequalities, and used measurements with continuous outcomes.

EPR-steering is a form of quantum non-locality that is logically intermediate ${ }^{2,15}$ between Bell non-locality ${ }^{19}$ and nonseparability. The second party, Bob, trusts quantum mechanics to describe his own measurements, but makes no assumptions about the distant first party, Alice, who has to convince him that she can affect the nature of his quantum state by her choice of measurement setting. So termed in analogy to Bell inequalities, EPR-steering inequalities ${ }^{14}$ are a superset of the former, and a subset of entanglement witnesses. Steering inequalities are, in principle, easier to violate experimentally than Bell inequalities because of the asymmetry between the parties; see Fig. 1. Instead of considering correlation functions for classical variables (measurement outcomes) on the two sides, in EPR-steering we consider correlations between classical variables declared by Alice but quantum expectation values measured by Bob.

Here we consider linear EPR-steering inequalities ${ }^{14}$ involving statistics collected from an experiment with $n$ measurement settings for each side. For qubits, we can take Bob's $k$ th measurement setting to correspond to the Pauli observable $\hat{\sigma}_{k}^{\mathrm{B}}$, along some axis $\mathbf{u}_{k}$. Denoting Alice's corresponding declared result (we make no assumption that it is derived from a quantum measurement) by the random variable $A_{k} \in\{-1,1\}$ for all $k$, the EPR-steering inequality is of the form

$$
S_{n} \equiv \frac{1}{n} \sum_{k=1}^{n}\left\langle A_{k} \hat{\sigma}_{k}^{\mathrm{B}}\right\rangle \leq C_{n}
$$

We call the quantity $S_{n}$ the steering parameter for $n$ measurement settings. The bound $C_{n}$ is the maximum value $S_{n}$ can have if Bob has a pre-existing state known to Alice, rather than half of an entangled pair shared with Alice. It is easy to see that this bound is

$$
C_{n}=\max _{\left\{A_{k}\right\}}\left\{\lambda_{\max }\left(\frac{1}{n} \sum_{k=1}^{n} A_{k} \hat{\sigma}_{k}^{\mathrm{B}}\right)\right\}
$$

where $\lambda_{\max }(\hat{O})$ denotes the largest eigenvalue of $\hat{O}$. 


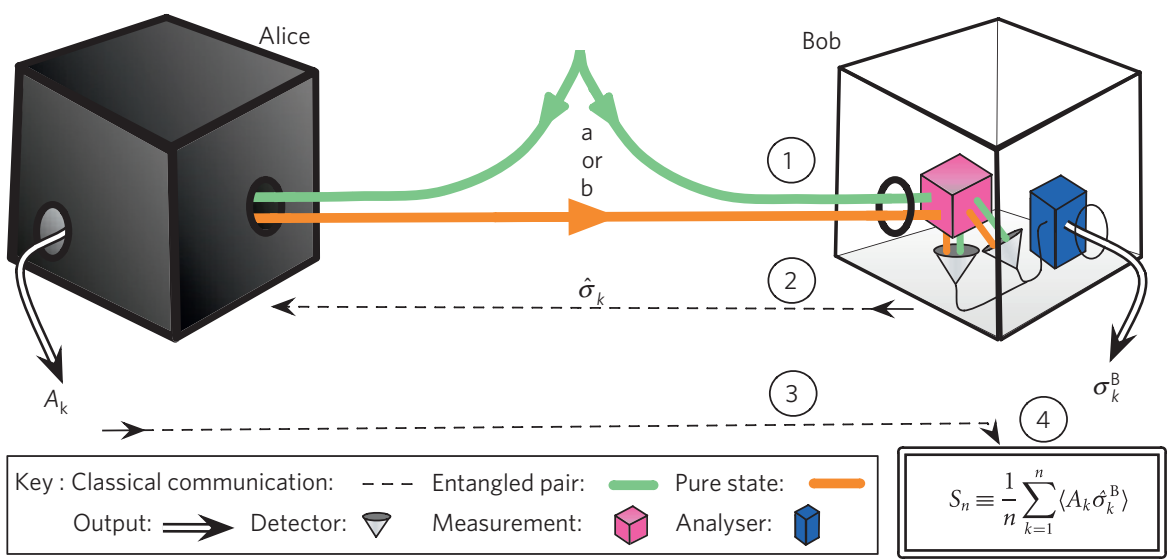

Figure 1 The steering task. Bob is sceptical that Alice can remotely affect (steer) his state. Bob trusts his measuring device (represented by the white box), in particular that it behaves according to the laws of quantum mechanics, but makes no assumptions about Alice's system and devices (represented by the black box). The steps in the task, from top (1) to bottom (4) are as follows. (1) Bob receives his qubit. He is unsure whether he has received (a) half of an entangled pair or (b) a pure state sent by Alice. (2) After Bob receives his qubit, he announces to Alice his choice of measurement setting from the set $\left\{\hat{\sigma}_{k}^{B}\right\}$. (3) Bob records his own measurement results $\sigma_{k}^{B}$ and receives the result $A_{k}$ that Alice declares. (4) Bob combines the results to calculate (over many runs) the steering parameter $S_{n}$. If this is greater than a certain bound, Alice has demonstrated steering of Bob's state, and thus Bob can be sure that he received $a$, not $b$.

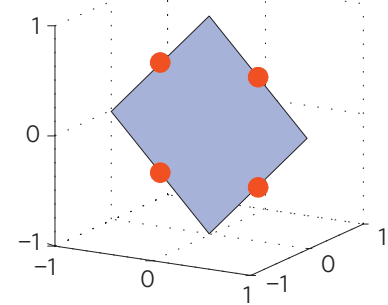

b

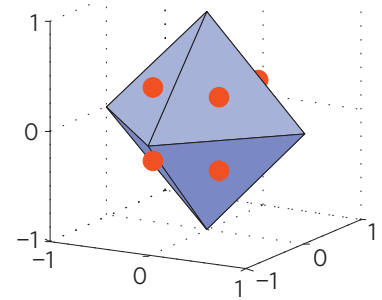

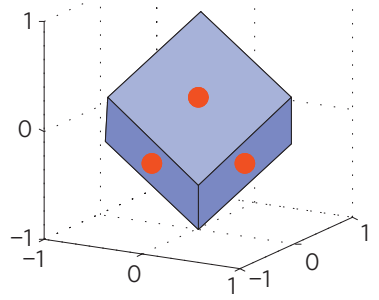

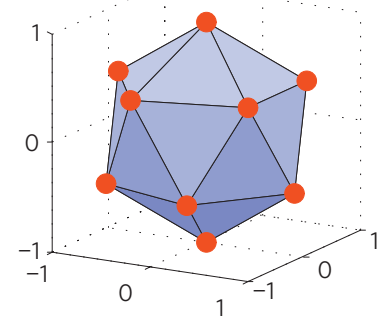

e

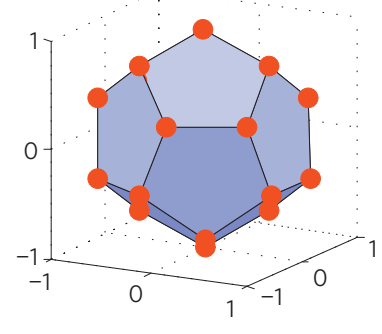

Figure 2 | Platonic-solid measurement schemes. Measurement axes $\mathbf{u}_{k}$ are defined by the Bloch-space directions through antipodal pairs of vertices of regular figures. a-e, Square, $n=2$ (a), and the four suitable Platonic solids: octahedron, $n=3$ (b); cube, $n=4$ (c); icosahedron, $n=6$ (d), and dodecahedron, $n=10$ (e). The bullet symbols show the orientations of pure states in optimal cheating ensembles for two-qubit Werner states. In $\mathbf{d}$ and $\mathbf{e}$ these states align with the measurement axes (vertices), but in a-c they have the dual arrangement, on the face centres, similar to the situation in random-access $\operatorname{codes}^{28}$.

To derive useful inequalities we consider measurement settings based around the four Platonic solids that have vertices that come in antipodal pairs (Fig. 2). Each pair defines a measurement axis $\mathbf{u}_{k}$, giving us an arrangement for $n=3$, 4, 6 and 10 settings. For $n=2$ settings, we use a square arrangement. For each measurement scheme (except for $n=10$, which we did not implement experimentally) we do the following: (i) Derive the bound $C_{n}$ in the inequality (1). (ii) Experimentally demonstrate EPR-steering by violating the inequality using Werner states. (iii) Theoretically show that Alice can saturate the inequality by sending Bob pure states drawn by her from a particular ensemble. (iv) Experimentally demonstrate (iii) above by nearly saturating the EPR-steering inequality in that way.
Werner states ${ }^{18}$ are the best-known class of mixed entangled states. For qubits, they can be written as

$$
W_{\mu}=\mu\left|\Psi^{-}\right\rangle\left\langle\Psi^{-}\right|+(1-\mu) \mathbf{I} / 4
$$

where $\left|\Psi^{-}\right\rangle$is the singlet state and I is the identity, and where $\mu \in[0,1]$. Werner states are entangled if and only if (iff) $\mu>1 / 3$ (ref. 18). They can violate the Clauser, Horne, Shimony and Holt ${ }^{20}$ (Bell-CHSH) inequality only if $\mu>1 / \sqrt{2}$, and cannot violate any Bell inequality if $\mu<0.6595$ (ref. 9). Reference 2 showed that these states are also steerable, with $n \rightarrow \infty$ settings, iff $\mu>1 / 2$. With $n=2$ projective measurements they are steerable iff $\mu>1 / \sqrt{2}$, no better than the Bell-CHSH inequality. Deriving analytical expressions for the bounds $C_{n}$ is a simple exercise in geometry (see Methods). 


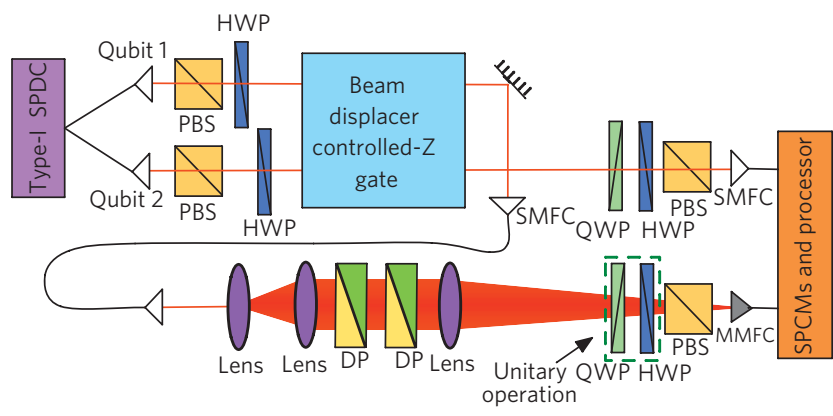

Figure 3 | Experimental set-up. Pairs of identical photons are produced using type-I SPDC and directed, by single-mode fibres, to a linear-optics controlled- $Z$ logic gate. After the gate, qubit 1 passes through a pair of Hanle wedge DPs. By varying the azimuthal angle between the optical axes of the DPs, we control the amount of depolarizing noise, which sets $\mu$ (see Methods). Abbreviations: SMFC, single-mode fibre coupler; MMFC, multi-mode fibre coupler.

For the square, octahedron and cube we find $C_{2}=1 / \sqrt{2}$ and $C_{3}=C_{4}=1 / \sqrt{3} \approx 0.5773$. For higher $n$ the exact expressions are lengthy; the approximate numerical values are $C_{6} \approx 0.5393$ and $C_{10} \approx 0.5236$. For a Werner-state experiment, the expected value of $S_{n}$ is $\mu$ (shown below). Thus, using $n \geq 3$ enables us to demonstrate EPR-steering for some Bell-local states, that is, states with $0.6595>\mu>1 / 2$. Also, with $n$ as small as $6, C_{n}$ is already within $8 \%$ of the $n \rightarrow \infty$ limit.

Consider the EPR-steering experiment, Fig. 1, from the point of view of an honest Alice, who does share a suitable entangled state with Bob. She claims to be able to prepare different types of state for Bob by making different remote measurements on her half of the state. If the state is a Werner state, she would claim to be able to prepare mixed states aligned (or anti-aligned) along any Bloch-sphere axis $\mathbf{u}$. They agree to test this along a specific set of axes $\left\{\mathbf{u}_{k}\right\}$. To maximize the correlation $S_{n}$ in equation (1), Alice measures $-\hat{\sigma}_{k}$, and announces her result $A_{k}$. The value of the correlation $S_{n}$ thus obtained will be $\mu$, independent of $n$, owing to the $\hat{U} \otimes \hat{U}$ invariance of the Werner state. Thus, for a given $n$, it should be possible to demonstrate EPR-steering if $\mu>C_{n}$.

We experimentally demonstrate EPR-steering with Werner states in a polarization-encoded two-qubit photonic system, as shown in Fig. 3 and detailed in the Methods section. In Fig. 4 we show data for a variety of Werner states; in each case we measured the Bell-CHSH parameter, $B$, following the method of ref. 21 , and the EPR-steering $S_{3}$ parameter. We clearly see states that violate both a Bell-CHSH inequality and an $S_{3}$ inequality, states that violate an $S_{3}$ inequality but not a Bell-CHSH inequality and states that violate neither inequality, but are still entangled.

The amount of entanglement required to demonstrate steering decreases as the number of equally spaced measurement axes increases, that is, for Platonic solids of increasing order (Fig. 2). We measure $S_{n}$ for states near the various steering bounds; see Fig. 5. We compare the values of $S_{2}$ and $S_{3}$ for each of three particular states. These states experimentally show that there exist cases where a state violates both the $S_{2}$ and $S_{3}$ inequalities, violates neither inequality or-most interestingly-violates the $S_{3}$ but not the $S_{2}$ inequality. Similar behaviour is observed for states near the $S_{4}$ and $S_{6}$ bounds. The $S_{3}$ and $S_{4}$ comparison is not especially interesting as $C_{3}=C_{4}$.

Now consider the EPR-steering experiment, Fig. 1, from the point of view of a dishonest Alice, who shares no entanglement with Bob. Such an Alice can adopt the following 'cheating' strategy. Draw a state $\left|\phi_{j}\right\rangle$ from some local-hidden-state (LHS) ensemble $E_{n}=\left\{\left|\phi_{j}\right\rangle\right\}$ and send it to Bob. Then, when Bob announces the measurement $\mathbf{u}_{k}$, announce a result $A_{k}(j)$ on the basis of the selected local hidden state and knowledge of Bob's measurement setting.

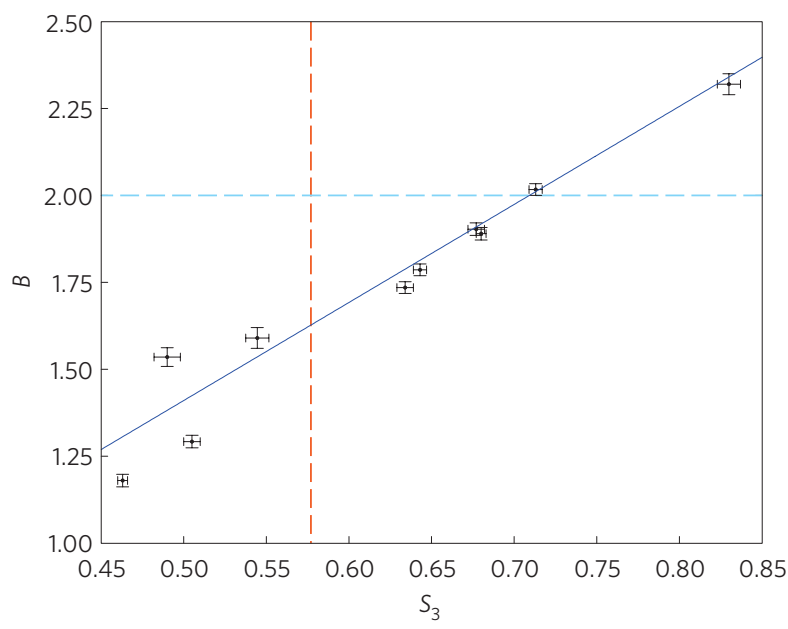

Figure 4 | Experimental demonstration of $S_{3}$ steering with Bell-local states. Data for tests of Bell non-locality (using the two-setting Bell-CHSH inequality) and EPR-steering using our three-setting inequality. The horizontal dashed line is the Bell-CHSH inequality bound, and the vertical dashed line is the $S_{3}$ bound. The diagonal blue line shows the predicted values for Werner states of varying $\mu$. Each data point corresponds to a different experimentally produced Werner state. Error bars represent one standard deviation, and are calculated from Poissonian counting statistics.

Although we call this a cheating strategy, Alice cannot actually cheat; the bound $C_{n}$ in equation (1) is defined exactly so that it is saturated by the optimal cheating LHS ensemble. That is, the bounds we have derived are tight; a value of $S_{n}$ greater than $C_{n}$ is necessary to demonstrate EPR-steering.

From the symmetry of Bob's measurement scheme, there are two obvious candidate LHS ensembles $E_{n}$ : the vertex ensemble and the dual ensemble. In the first case the states $\left|\phi_{j}\right\rangle$ are oriented on the Bloch sphere in the directions of the vertices of the figure defining $\left\{\mathbf{u}_{k}\right\}$. In the second, they are oriented in the direction of the face centres (that is, the vertices of the dual figure). Interestingly, both of these possibilities are optimal, but for different values of $n$; see Fig. 2 . Given an optimal ensemble, Alice's optimal 'cheating' strategy, having been told Bob's measurement axis $\mathbf{u}_{k}$, is to announce as $A$ the more likely outcome $(+1$ or -1$)$ of Bob's measurement on the state $\left|\phi_{j}\right\rangle$ she has sent.

The experimental realization is simple-Alice prepares a single qubit state using a polarizing beam splitter (PBS), a half-wave plate (HWP) and a quarter-wave plate (QWP), and this state is measured by Bob as before. We experimentally demonstrate the near-saturation of the bound $C_{n}$ using the optimal cheating ensemble for Alice, achieving above $95 \%$ saturation for all tested $C_{n}$, as shown in Fig. 5. The small discrepancy from perfect saturation is due to imperfect state preparation and measurement, even though the prepared states were tomographically measured to overlap with the ideal states to $>99 \%$ fidelity. The discrepancy increases with $n$ as the cumulative effect of slight misalignments in a series of measurements or preparations tends to reduce the observed $C_{n}$.

Our demonstration of EPR-steering using states that violate no Bell inequality is possible only because we have broken the conceptual shackles of previous EPR experiments ${ }^{3-6}$. These followed the approach of ref. 7 based on the uncertainty relation for two observables with continuous spectra. We used discrete measurements on entangled qubits, and used up to $n=6$ measurement settings, showing that that increasing $n$ makes the EPR-steering inequality much more robust to noise. In our work we made the fair-sampling assumption, that undetected photons are statistically identical to detected photons. Thus we were content with an experimental efficiency far below that necessary 

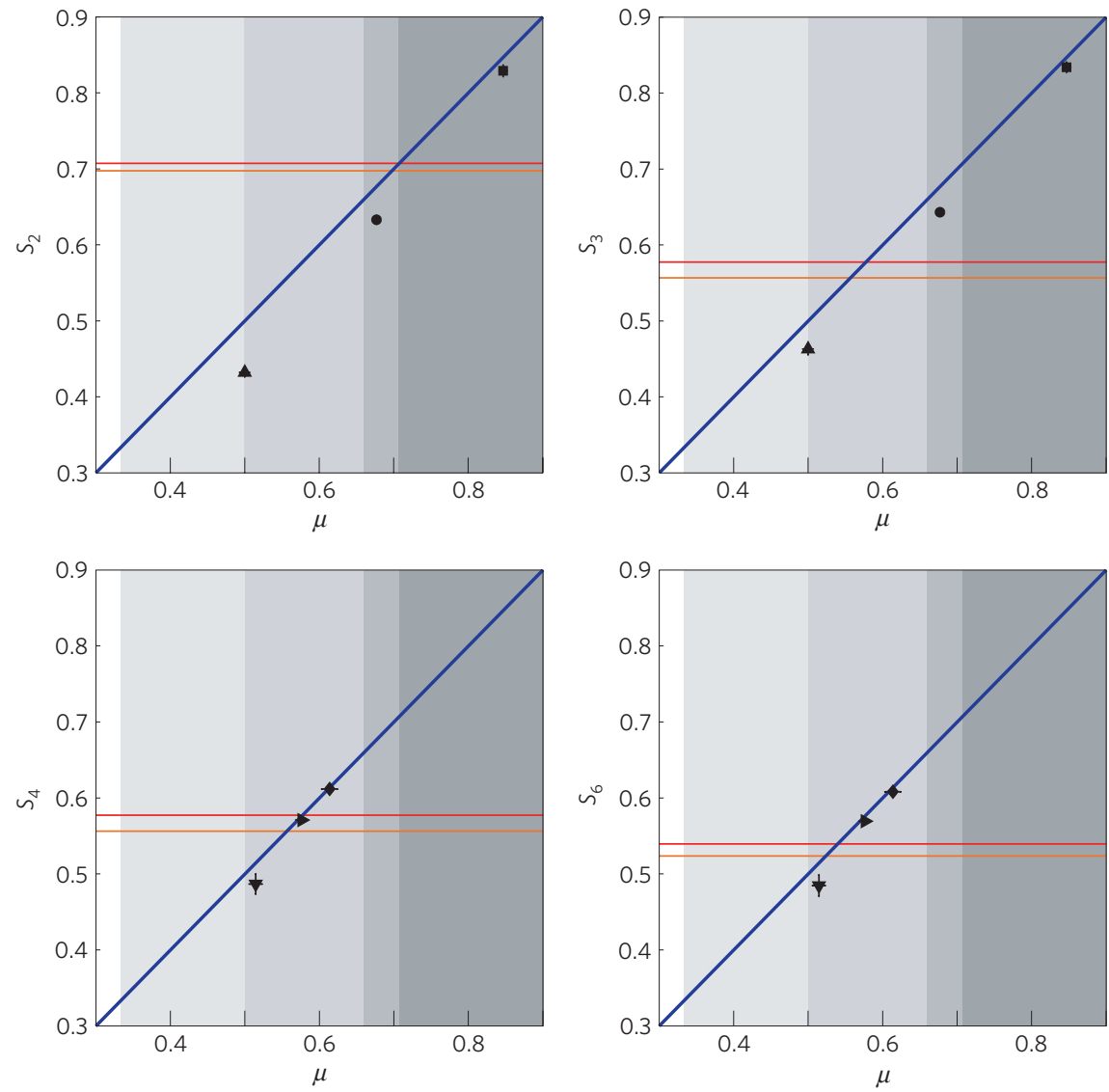

Figure 5 | EPR-steering and 'cheating' strategies for increasing $\boldsymbol{n}$. The four plots show the measured EPR-steering correlation $S_{n}$ for various Werner states against their tomographically reconstructed values of $\mu$, for $n=2,3,4$ and 6 . The blue diagonal line in each plot represents the theoretical value of $S_{n}$. The vertical shaded regions represent different entanglement classes for Werner states. Left to right: separable; non-separable but not steerable (even for $n \rightarrow \infty$ ); steerable but not Bell non-local; potentially Bell non-local; Bell non-local by the simple Bell-CHSH test. (For 0.7071 > $\mu>0.7056$, there is a positive Bell-non-locality test using 465 settings a side ${ }^{11}$.) The red solid horizontal line represents the $S_{n}$ theoretically attainable by a 'cheating' strategy by Alice. This is the face-centred ensemble, except for $n=6$, where it is the vertex ensemble. This defines the steering bound $C_{n}-$ EPR-steering is demonstrated if $S_{n}$ exceeds this. As in Fig. 1, the orange solid line (slightly below $C_{n}$ ) represents the experimentally attained value using the optimal cheating ensemble. The square highlights a state that is both steerable for $n=2$ and $n=3$ and also Bell non-local. The circle and right-pointing triangle show states that become steerable as $n$ increases, from $n=2$ to $n=3$ and $n=4$ to $n=6$ respectively. The diamond is a state that is steerable for two different measurement schemes and is Bell local. The up-pointing triangle is not steerable, whereas the down-pointing triangle is steerable in principle but could not be steered using any of the finite- $n$ inequalities implemented by us. Error bars (shown only when large enough to be clearly seen) are one standard deviation and are calculated from Poissonian counting statistics.

to demonstrate EPR-steering without this assumption. However, because the degree of correlation required for EPR-steering is smaller than that for violation of a Bell inequality, it should be correspondingly easier to demonstrate steering of qubits without making the fair-sampling assumption. This would provide an important and exciting extension of the fundamental principles we have demonstrated, and open the door to the application of EPR-steering phenomena for quantum communication protocols.

\section{Methods}

Werner-state production. To realize the Werner states, we start by generating identical single photons through type-I spontaneous parametric downconversion (SPDC). These photon pairs are initially unentangled in polarization. We use a non-deterministic controlled- $Z$ gate ${ }^{22-25}$ to entangle them in polarization. Ideally, this creates the state $\left(H_{1} \otimes \mathbf{I}\right)\left|\Psi^{-}\right\rangle$, where $H_{1}$ is the Hadamard gate ${ }^{22}$ acting on qubit 1. Mixture was controllably added, enabling a change of $\mu$, using the depolarizer (DP) method of Puentes et al. ${ }^{26}$ - see Fig. 3 and description below. This method produces 'Werner-like' states-states equivalent up to local rotations to the Werner states of equation (3).

The Werner-like states are described by $\rho=(\hat{U} \otimes \mathbf{I}) W_{\mu}(\hat{U} \otimes \mathbf{I})^{\dagger}$, where $\hat{U}$ is a single-qubit unitary operation. We can undo $\hat{U}$ to retrieve a Werner state by incorporating a unitary transformation in the measurement settings of qubit 1 . To find the optimal unitary operation, we first tomographically reconstruct $\rho$ following ref. 27. We numerically search for $\hat{U}$ by maximizing the fidelity of $W_{\mu}$ with $(\hat{U} \otimes \mathbf{I}) \rho\left(\hat{U}^{\dagger} \otimes \mathbf{I}\right)$. The maximizing $\hat{U}$ is then used to rotate the measurement settings for qubit 1 .

Photon source and controlled- $Z$ gate. Source: a $60 \mathrm{~mW}$, linearly polarized, continuous-wave 410-nm-wavelength laser is used to pump a BiBO (bismuth borate) nonlinear crystal to produce pairs of $820 \mathrm{~nm}$ single photons through type-I SPDC. With a coincidence window of $3 \mathrm{~ns}$, a coincidence rate of approximately 10,000 counts s ${ }^{-1}$ is achieved. Controlled- $Z$ gate: the gate is implemented using a passively stable beam displacer configuration, as in ref. 22 . We are not concerned with the gate's limited success probability in generating entangled states, as we make the fair-sampling assumption. The effective efficiency (coincidences-to-singles ratio) is $\approx 0.2 \%$ in this experiment.

Depolarizer method. By varying the azimuthal angle between two quartz-glass Hanle $\mathrm{DPs}^{26}$, we create a tunable, variable depolarizing device. It couples the polarization degree of freedom to the spatial degree of freedom-tracing over spatial information induces mixture. By optimizing these procedures, high-quality Werner states (fidelity $\geqslant 93.5 \%$ in each case) were produced for a wide range of $\mu$.

Measurement technique. We implement single-qubit polarization measurements on each qubit using a QWP, an HWP, a PBS and fibre-coupled single-photon counting modules (SPCMs), enabling us to measure along arbitrary axes on the Bloch sphere for each qubit. By choosing different combinations of measurement 
axes, we can carry out a variety of measurement tasks: evaluating the Bell-CHSH inequality, evaluating the steering parameter $S_{n}$ for different $n$ or tomographically reconstructing the Werner-like states, $\rho$.

Calculating $C_{n}$. In each case we search over the possible sets $\left\{A_{k}\right\}$, numerically evaluating the maximand in equation (2). Then, choosing one of the sets that attains the maximum, we use the geometry of the relevant Platonic solid to evaluate it analytically. The same analytical expressions are found from the optimal LHS ensembles $E_{n}$ of Fig. 2. Those not given in the main text are:

$$
\begin{gathered}
C_{6}=1-\left(5 L_{6} / 12\right) \sqrt{4-\sec ^{2}(3 \theta / 2)} \\
C_{10}=1-\frac{1}{10}\left(1+\frac{\tan 2 \theta}{\sin \theta}\right) \sqrt{9 L_{10}^{2}-4}
\end{gathered}
$$

Here $L_{6}=4 / \sqrt{10+2 \sqrt{5}}$ and $L_{10}=4 /(\sqrt{15}+\sqrt{3})$ are the side lengths of an icosahedron and dodecahedron respectively, circumscribed by the Bloch sphere, and $\theta=\pi / 5$.

Received 11 May 2010; accepted 6 August 2010; published online 19 September 2010; corrected after print 2 November 2011

\section{References}

1. Schrödinger, E. Discussion of probability relations between separated systems. Proc. Camb. Phil. Soc. 31, 555-563 (1935).

2. Wiseman, H. M., Jones, S. J. \& Doherty, A. C. Steering, entanglement, nonlocality, and the Einstein-Podolsky-Rosen paradox. Phys. Rev. Lett. 98, 140402 (2007)

3. Ou, Z. Y., Pereira, S. F., Kimble, H. J. \& Peng, K. C. Realization of the Einstein-Podolsky-Rosen paradox for continuous variables. Phys. Rev. Lett. 68, 3663-3666 (1992).

4. Bowen, W. P., Schnabel, R., Lam, P. K. \& Ralph, T. C. Experimental investigation of criteria for continuous variable entanglement. Phys. Rev. Lett. 90, 043601 (2003).

5. Hald, J., Sørensen, J. L., Schori, C. \& Polzik, E. S. Spin squeezed atoms: A macroscopic entangled ensemble created by light. Phys. Rev. Lett. 83, 1319-1322 (1999).

6. Howell, J. C., Bennink, R. S., Bentley, S. J. \& Boyd, R. W. Realization of the Einstein-Podolsky-Rosen paradox using momentum- and position-entangled photons from spontaneous parametric down conversion. Phys. Rev. Lett. 92, 210403 (2004)

7. Reid, M. D. Demonstration of the Einstein-Podolsky-Rosen paradox using nondegenerate parametric amplification. Phys. Rev. A 40, 913-923 (1989)

8. Altepeter, J. B. et al. Experimental methods for detecting entanglement. Phys. Rev. Lett. 95, 033601 (2005)

9. Acìn, A., Gisin, N. \& Toner, B. Grothendieck's constant and local models for noisy entangled quantum states. Phys. Rev. A 73, 062105 (2006).

10. Brunner, N. \& Gisin, N. Partial list of bipartite Bell inequalities with four binary settings. Phys. Lett. A 372, 3162-3167 (2008).

11. Vértesi, T. More efficient Bell inequalities for Werner states. Phys. Rev. A 78, $032112(2008)$.
12. Einstein, A., Podolsky, B. \& Rosen, N. Can quantum-mechanical description of physical reality be considered complete? Phys. Rev. 47, 777-780 (1935).

13. Reid, M. D. et al. Colloquium: The Einstein-Podolsky-Rosen paradox: From concepts to applications. Rev. Mod. Phys. 81, 1727-1751 (2009).

14. Cavalcanti, E. G., Jones, S. J., Wiseman, H. M. \& Reid, M. D. Experimental criteria for steering and the EPR paradox. Phys. Rev. A. 80, 032112 (2009).

15. Jones, S. J., Wiseman, H. M. \& Doherty, A. C. Entanglement, Einstein-Podolsky-Rosen correlations, Bell nonlocality, and steering. Phys. Rev. A 76, 052116 (2007).

16. White, A. G. et al. Measuring two-qubit gates. J. Opt. Soc. Am. B 24, 172-183 (2007).

17. Barrett, J. Nonsequential positive-operator-valued measurements on entangled mixed states do not always violate a Bell inequality. Phys. Rev. A 65, 042302 (2002).

18. Werner, R. F. Quantum states with Einstein-Podolsky-Rosen correlations admitting a hidden-variable model. Phys. Rev. A 40, 4277-4281 (1989).

19. Bell, J. S. On the Einstein-Podolsky-Rosen paradox. Physics (Long Island City, N.Y.) 1, 195-200 (1964).

20. Clauser, J. F., Horne, M. A., Shimony, A. \& Holt, R. A. Proposed experiment to test local hidden-variable theories. Phys. Rev. Lett. 23, 880-884 (1969).

21. Kwiat, P. G., Waks, E., White, A. G., Appelbaum, I. \& Eberhard, P. H. Ultrabright source of polarization-entangled photons. Phys. Rev. A 60, R773-R776 (1999).

22. O'Brien, J. L. et al. Demonstration of an all-optical quantum controlled-NOT gate. Nature 426, 264-267 (2003).

23. Langford, N. K. et al. Demonstration of a simple entangling optical gate and its use in Bell-state analysis. Phys. Rev. Lett. 95, 210504 (2005).

24. Kiesel, N. et al. Linear optics controlled-phase gate made simple. Phys. Rev. Lett. 95, 210505 (2005)

25. Okamoto, R., Hofmann, H. F., Takeuchi, S. \& Sasaki, K. Demonstration of an optical quantum controlled-not gate without path interference. Phys. Rev. Lett. 95, 210506 (2005).

26. Puentes, G., Voigt, D., Aiello, A. \& Woerdman, J. P. Tunable spatial decoherers for polarization-entangled photons. Opt. Lett. 31, 2057-2059 (2006).

27. James, D. F. V., Kwiat, P. G., Munro, W. J. \& White, A. G. Measurement of qubits. Phys. Rev. A 64, 052312 (2001).

28. Spekkens, R. W. et al. Preparation contextuality powers parity-oblivious multiplexing. Phys. Rev. Lett. 102, 010401 (2009).

\section{Acknowledgements}

This work was supported by the Australian Research Council. We thank E. Cavalcanti, A. Fedrizzi, D. Kielpinski, and A. G. White for discussions. We also thank B. Higgins, M. Palsson and S. Kocsis for their contributions.

\section{Author contributions}

H.M.W. conceived the theory. S.J.J. and H.M.W. developed the theory. G.J.P. developed the experiment. H.M.W. and G.J.P. supervised the project. D.J.S. built the experiment, and collected and analysed the data. D.J.S., S.J.J., H.M.W. and G.J.P. composed the manuscript.

\section{Additional information}

The authors declare no competing financial interests. Reprints and permissions information is available online at http://npg.nature.com/reprintsandpermissions. Correspondence and requests for materials should be addressed to H.M.W. or G.J.P. 


\section{CORRIGENDUM}

\section{Experimental EPR-steering using Bell-local states}

\section{J. Saunders, S. J. Jones, H. M. Wiseman and G. J. Pryde}

Nature Physics 6, 845-849 (2010); published online: 19 September 2010; corrected after print: 2 November 2011.

The authors wish to point out that there were systematic errors in some of the demonstrations of Alice's optimal attempt to cheat (orange lines in Fig. 5, for $n=3,4$ and 6), due to misalignment of the waveplates. The data have been retaken with the corrected settings and are included in the following corrected figure (the plot for $n=2$ is unchanged). The arguments of the paper are unaffected by this correction. These changes have been made in the PDF and HTML versions of this Letter.
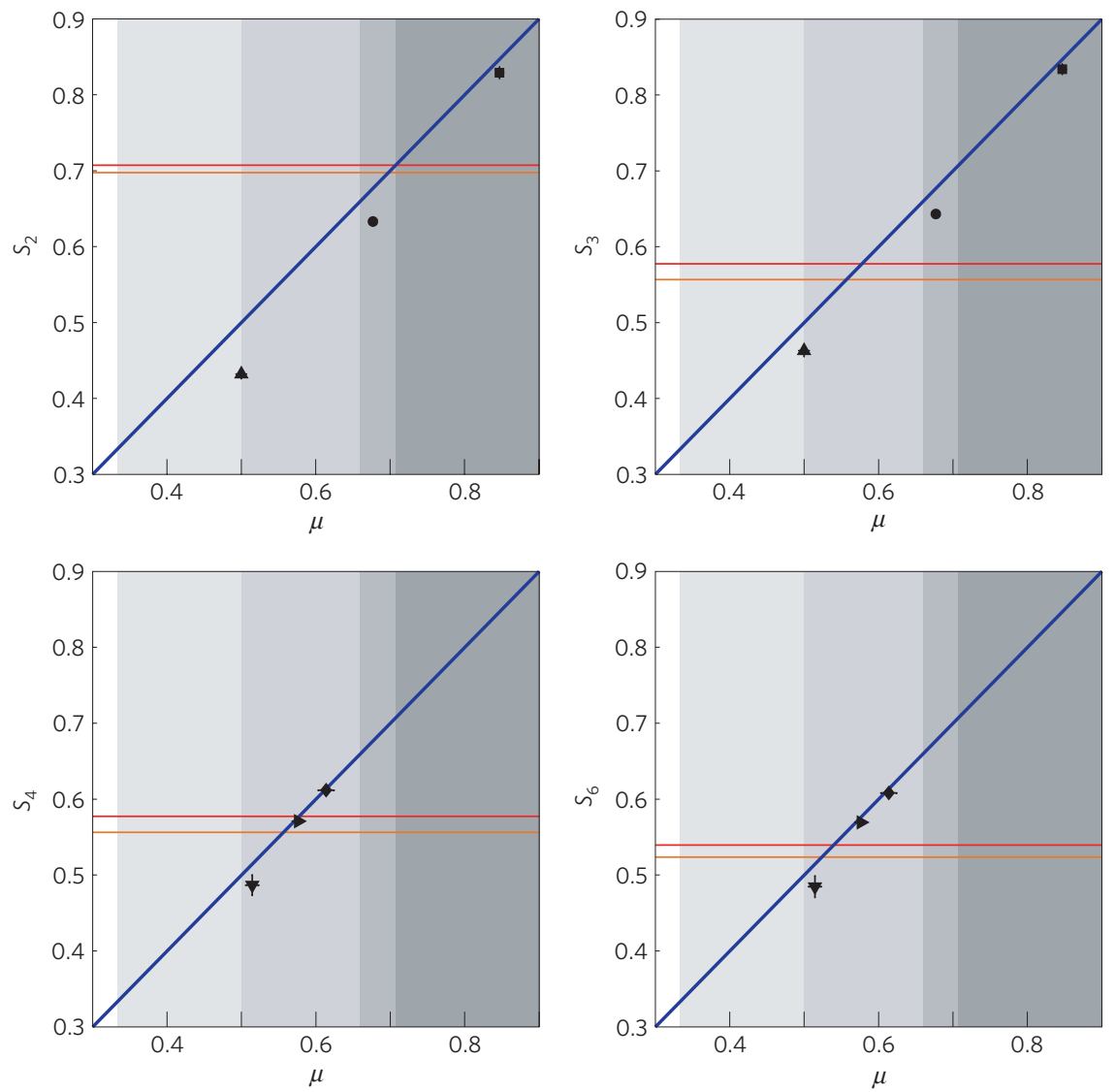